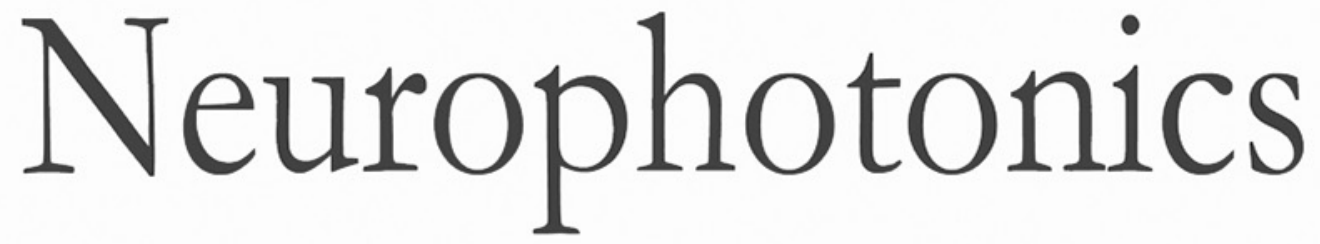

\title{
Transcranial photobiomodulation with near-infrared light from childhood to elderliness: simulation of dosimetry
}

\author{
Yaoshen Yuan \\ Paolo Cassano \\ Matthew Pias \\ Qianqian Fang
}




\title{
Transcranial photobiomodulation with near-infrared light from childhood to elderliness: simulation of dosimetry
}

\author{
Yaoshen Yuan, ${ }^{a}$ Paolo Cassano,,${ }^{\text {b,c }}$ Matthew Pias, ${ }^{d}$ and Qianqian Fang ${ }^{d, a, *}$ \\ ${ }^{\mathrm{a} N o r t h e a s t e r n ~ U n i v e r s i t y, ~ D e p a r t m e n t ~ o f ~ E l e c t r i c a l ~ a n d ~ C o m p u t e r ~ E n g i n e e r i n g, ~ B o s t o n, ~}$ \\ Massachusetts, United States \\ ${ }^{b}$ Massachusetts General Hospital, Depression Clinical and Research Program, Center for \\ Anxiety and Traumatic Stress Disorders, Boston, Massachusetts, United States \\ ${ }^{c}$ Harvard Medical School, Department of Psychiatry, Boston, Massachusetts, United States \\ ${ }^{\mathrm{d} N o r t h e a s t e r n ~ U n i v e r s i t y, ~ D e p a r t m e n t ~ o f ~ B i o e n g i n e e r i n g, ~ B o s t o n, ~ M a s s a c h u s e t t s, ~}$ \\ United States
}

\begin{abstract}
Significance: Major depressive disorder (MDD) affects over 40 million U.S. adults in their lifetime. Transcranial photobiomodulation (t-PBM) has been shown to be effective in treating MDD, but the current treatment dosage does not account for head and brain anatomical changes due to aging.
\end{abstract}

Aim: We study effective t-PBM dosage and its variations across age groups using state-of-the-art Monte Carlo simulations and age-dependent brain atlases ranging between 5 and 85 years of age.

Approach: Age-dependent brain models are derived from 18 MRI brain atlases. Two extracranial source positions, F3-F4 and Fp1-Fpz-Fp2 in the EEG 10-20 system, are simulated at five selected wavelengths and energy depositions at two MDD-relevant cortical regions-dorsolateral prefrontal cortex (dIPFC) and ventromedial prefrontal cortex (vmPFC) - are quantified.

Results: An overall decrease of energy deposition was found with increasing age. A strong negative correlation between the thickness of extracerebral tissues (ECT) and energy deposition was observed, suggesting that increasing ECT thickness over age is primarily responsible for reduced energy delivery. The F3-F4 position appears to be more efficient in reaching dIPFC compared to treating vmPFC via the Fp1-Fpz-Fp2 position.

Conclusions: Quantitative simulations revealed age-dependent light delivery across the lifespan of human brains, suggesting the need for personalized and age-adaptive t-PBM treatment planning.

(C) The Authors. Published by SPIE under a Creative Commons Attribution 4.0 Unported License. Distribution or reproduction of this work in whole or in part requires full attribution of the original publication, including its DOI. [DOI: 10.1117/1.NPh.7.1.015009]

Keywords: transcranial photobiomodulation; Monte Carlo methods; optical dosimetry; major depressive disorder.

Paper 19094R received Sep. 22, 2019; accepted for publication Feb. 5, 2020; published online Feb. 24, 2020.

\section{Introduction}

According to the U.S. National Institutes of Health, ${ }^{1}$ the estimated lifetime prevalence of major depressive disorder (MDD) in the United States comprises over 13\% of the population. MDD can develop at any age and is considered the leading cause of disability in the U.S. for individuals between the ages of 15 and 44 years. $^{2-4}$ The two most commonly used treatments for MDD are antidepressants $(87.0 \%)$ and psychotherapy $(23.2 \%){ }^{5}$ Several known challenges have been faced by these treatment approaches: (1) frequent relapses of the cognitive therapy ${ }^{6}$ and

*Address all correspondence to Qianqian Fang, E-mail: q.fang@neu.edu 
(2) burdensome side effects of antidepressant medication. ${ }^{7}$ Furthermore, many patients prefer to self-manage, which leads to the low treatment rates. ${ }^{8}$ Therefore, new, effective, safe, and easy-toadminister treatment methods are needed to battle MDD.

Photobiomodulation (PBM) is a near-infrared (NIR) light-based therapy technique and has shown therapeutic effectiveness for various neuropsychiatric disorders, including MDD. ${ }^{9-11}$ The transcranial PBM (t-PBM) technique delivers NIR light through the scalp and skull. ${ }^{12-14}$ Due to the penetration depth of NIR light in human tissues, clinically effective light dosages can be delivered to the disease-responsible brain regions without damaging superficial tissues. Although the molecular mechanisms of PBM remain a topic of active research, some studies report that treatment effects may derive from the excitation of a mitochondrial chromophorecytochrome c oxidase - at the NIR spectra ${ }^{14}$ stimulating the mitochondrial respiratory chain and increasing adenosine triphosphate production. ${ }^{10,11}$ The concurrent upshot of reactive oxygen species may trigger cytoprotective and antioxidation pathways within the cell, with effects potentially lasting on the scale of days to weeks. ${ }^{15} \mathrm{~A}$ wide range of studies, in both animal models and humans, have shown that PBM causes minimal or no adverse effects while producing therapeutic effects. ${ }^{10,16,17}$

Although MDD has a broad age of onset, ${ }^{2,4}$ most previously published studies have focused on t-PBM treatments in only middle-aged adult brain models. ${ }^{18}$ However, personalization of treatments is key to increasing success rate and tolerability; therefore, our interest is in developing precise PBM treatment strategies adapted for individual patients. One of the main factors impacting t-PBM light dosage is the thickness of extracerebral tissues (ECTs), including both skull and scalp. ${ }^{19-21}$ Therefore, a quantitative analysis on how brain development and senescence could impact the effective dosage in a t-PBM treatment can provide valuable guidelines for clinicians to optimize their procedures and maximize treatment efficacy and tolerability.

To capture the variations of anatomical features among age groups, we have to first create anatomically appropriate brain/full-head models, including skin/skull/brain three-dimensional shapes and thicknesses. Fortunately, a number of recent studies have published comprehensive magnetic resonance imaging (MRI) atlases outlining the development of human brains from infants to elders. ${ }^{22-25}$ In addition, several groups, including our own, have developed sophisticated brain segmentation and meshing pipelines to convert neuroanatomical scans into highquality multilayered brain models. These resources make it possible to quantitatively investigate how the development and senescence of the human brain influences light penetration at different stages of life.

In addition, advanced photon transport models must be used to accurately account for the complex light-tissue interactions during t-PBM procedures. In this study, we applied the Monte Carlo (MC) method - a stochastic solver for the radiative transfer equation (RTE) — which is widely considered the gold standard for light modeling in complex tissues. ${ }^{26}$ While alternative models, such as the diffusion equation (DE), are dramatically faster and applicable to many types of human tissues, ${ }^{27,28}$ for brain tissues, DE is known to produce erroneous solutions due to the presence of low-scattering media, such as air cavities and cerebrospinal fluid (CSF). ${ }^{29,30}$ The MC method solves the RTE rigorously by simulating large numbers of photons following a set of known probability models derived from physics. ${ }^{31}$ The only major limitation is that MC methods are computationally expensive. To improve computational efficiency, we applied our widely disseminated hardware-accelerated MC modeling platform-Monte Carlo eXtreme (MCX). ${ }^{32}$ This tool can shorten the simulation runtime by several hundred times compared to conventional CPU-based simulations. ${ }^{32,33}$

The rest of the paper is organized as follows. In Sec. 2, we detail the preprocessing steps to create four-layer head segmentations from the neurodevelopmental MRI brain atlas library. ${ }^{22}$ We also report the steps to obtain brain parcellations and the placement of light source positions. In Sec. 3, the simulated energy depositions for $2 \mathrm{t}-\mathrm{PBM}$ source placements and 5 selected wavelengths on 18 selected brain/head atlases, ranging between 5 and 89 years of age are reported. In Sec. 4, we highlight the findings regarding the efficiency of different wavelengths, the energy deposition, and the exposure duration in the wide span of age groups. In addition, we also correlate our findings with the anatomical changes associated with the brain development and senescence. 


\section{Materials and Methods}

\subsection{Creating Multilayer Head Models from MRI Brain Atlas Library}

Brain segmentations are created by processing the Neurodevelopmental MRI database. ${ }^{22-24} \mathrm{We}$ select a total of 18 age groups, ranging from 5 through 89 years of age. Specifically, the average atlas for age groups $5,10,14,18,20$ to 24,25 to 29,30 to 34,35 to 39,40 to 44,45 to 49,50 to 54,55 to 59,60 to 64,65 to 69,70 to 74,75 to 79,80 to 84 , and 85 to 89 are used in our study. For each atlas, a four-layer full-head segmentation is created, including the white matter (WM), gray matter (GM), CSF, and ECT. An additional air cavity segmentation is created to properly model light propagation inside the nasal and pharyngeal cavities. As a result, a total of five tissue labels are considered. Three samples of segmented brain volumes at 5, 40 to 44 , and 85 to 89 years of age are shown in Figs. 1(a)-1(c), respectively.

The brain atlas probabilistic tissue segmentations (PTSs) provided in the Neurodevelopmental MRI database were derived from averaging subjects in each age group. The GM/WM volumes directly calculated from the atlas PTS volumes using a simple threshold show discrepancies compared to the GM/WM volumes estimated from the original group-based data published by the same authors. ${ }^{23,24}$ We believe that this discrepancy results from the averaging and nonlinear effects of the atlas creation process.

To correct for this discrepancy, an adaptive threshold $(T \in[0,1])$ is applied to the PTSs of WM, GM, and CSF of all atlases. As shown in Fig. 1(d), before this correction, a uniform threshold $T=0.5$ of the atlas segmentation (dashed lines) appears to underestimate the GM volume and overestimate the WM volume along age compared to the previously reported averaged volumes of the population ${ }^{23,24}$ from which the atlases are derived. To reduce this artifact, we dynamically estimate a threshold for GM/WM to match the tissue volumes to the population-derived estimations. The corrected GM/WM volumes (solid lines) over age are shown in Fig. 1(d). In comparison, such discrepancies for the CSF layer are relatively small compared

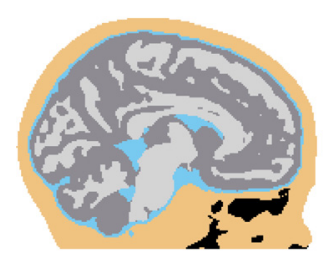

(a)

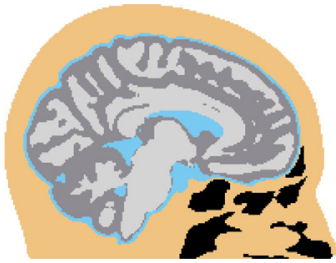

(b)

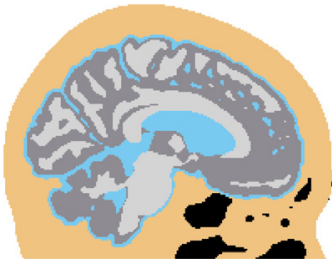

(c)

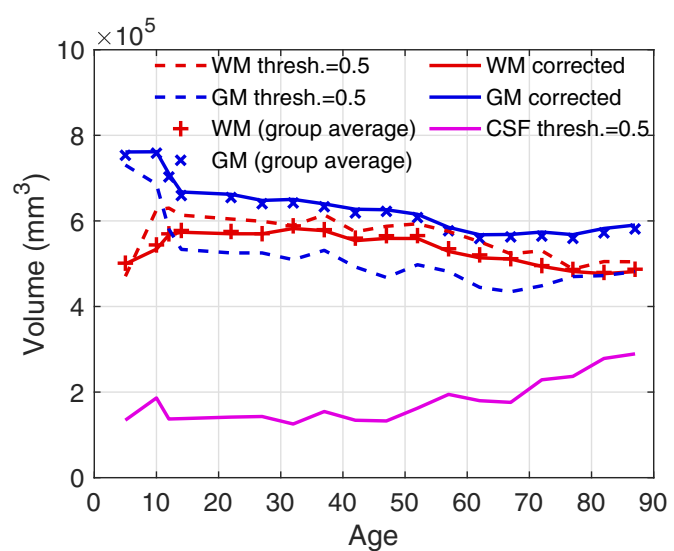

(d)

Fig. 1 Sample segmented brain models for (a) 5, (b) 40 to 44, and (c) 85 to 89 years old to visualize brain development. Black regions represent air cavities. (d) The WM, GM, and CSF volumes before (dashed lines) and after (solid lines) adaptive thresholding. The group-average WM and GM volumes derived from the source population are based on the studies of Fillmore et al. ${ }^{23}$ and Sanchez et al. ${ }^{24}$ 
to previous studies. ${ }^{23,24,34,35}$ For simplicity, a uniform threshold $T_{\mathrm{CSF}}=0.5$ is applied to T1weighted (T1w) CSF PTS.

To obtain the exterior surface of the ECT layer, i.e., the scalp surface, we use FMRIB Software Library (FSL) and the "betsurf" add-on to process the T1w MRI images provided by the atlas database. ${ }^{23,36}$ It is worth noting that the FSL pipeline for segmenting ECT tissues has been validated in adult brains/heads. For young-age children, only limited studies have been reported. ${ }^{37-39}$ Therefore, our results for young children are intended for qualitative assessment only.

\subsection{Segmentations of Air Cavities in the Brain Atlases}

It is important to note that the presence of air cavities in the brain atlases has significant impact to light dosimetry due to the low absorption of air. However, most neuroanatomical analysis tools do not have the ability to automatically extract these cavities. Here, we use a combination of manual segmentation and clustering analysis to extract various head cavities.

The frontal sinus and sphenoid sinus are manually segmented using the T1w MRI images and ITK-SNAP. ${ }^{40}$ To segment the nasal and pharyngeal cavities, we apply the $k$-means algorithm to the raw T1w MRI images. ${ }^{41}$ A total of three clusters are segmented for the ECT layer below Fpz point and the cluster with the lowest intensity is used as the air cavities. The eyes and the spine also appear as low-intensity regions in T1w images. A manual flood-filling operation is performed to identify these regions. Examples of the created multilayer brain anatomies are shown in Fig. 2(a).

\subsection{Brain Parcellations and Target Regions}

Similar to our previous study, ${ }^{18}$ our primary interest is to develop effective PBM treatment approaches for emotion regulation and depression. Thus, our main focuses in this dosimetry study are the dorsolateral prefrontal cortex (dIPFC) and ventromedial prefrontal cortex (vmPFC) regions [Fig. 2(b)], both involved in the emotion regulation circuitry. However, our approach is general and can be used to characterize all brain functional regions. We used the MarsAtlas

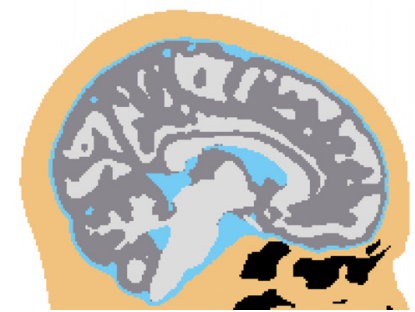

(a)

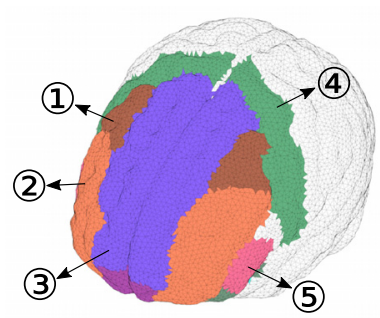

(c)

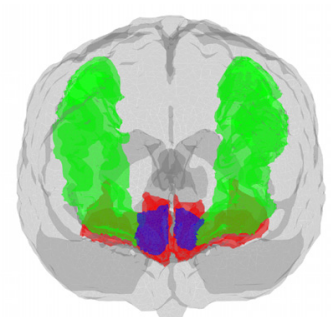

(b)

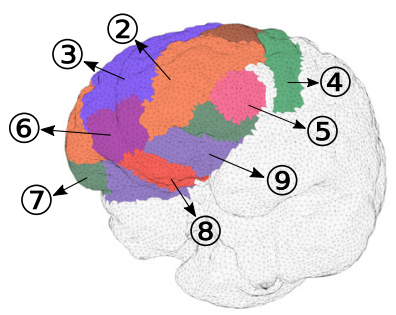

(d)

Fig. 2 Sample brain segmentation and ROI. (a) 18-0 years old four-layer model, including WM, GM, CSF, and ECT. Black regions indicate air cavities. (b) Green regions are dIPFC and red regions are vmPFC. The frontal pole is indicated by blue color. (c) and (d) Parcellations (bilateral) used in this study, including 1-caudal middle frontal gyrus, 2-rostral middle frontal gyrus, 3superior frontal gyrus, 4-precentral gyrus, 5-pars triangularis, 6-frontal pole, 7-pars orbitalis, 8-medial orbitofrontal cortex, and 9-lateral orbitofrontal cortex. 
parcellation in our previous study. ${ }^{18,42}$ This parcellation of the vmPFC region includes the frontal pole (Brodmann Area 10), whereas several other studies did not. ${ }^{43-46}$ This may potentially cause a discrepancy in calculations of energy deposition in vmPFC. In this study, we consider both definitions by selecting and merging subregions from the Desikan-Killiany-Tourville (DKT) parcellation ${ }^{47}$ that best comprises the vmPFC regions in either definition. The brain DKT parcellation is obtained using the "recon-all" workflow provided in FreeSurfer v6.0 ${ }^{48,49}$ [see Figs. 2(b)-2(d)]. The FreeSurfer brain parcellation workflow has been validated in previous publications for ages greater than 3 years of age. ${ }^{45,50}$

In this study, dIPFC is considered to cover two regions in the DKT parcellation-caudal middle frontal gyrus and rostral middle frontal gyrus [labels 1 and 2 in Fig. 2(c)] according to Ehrlich et al. ${ }^{51}$ The superior frontal gyrus is excluded because it is not relevant to t-PBM. As mentioned earlier, a general consensus on the boundary of the vmPFC has not been reached. In previous literature, vmPFC is either defined as the combination of (1) medial orbitofrontal cortex and lateral orbitofrontal cortex ${ }^{45,46}$ [labels 8 and 9 in Fig. 2(d)] or (2) frontal pole, medial orbitofrontal cortex, and lateral orbitofrontal cortex [labels 6, 8, and 9 in Fig. 2(d)], referred to as the extended vmPFC ${ }^{52,53}$ hereinafter. Furthermore, due to the slight mismatch between the FreeSurfer parcellations and the GM in our four-layer segmentation, the final parcellations are defined as the intersection between the two models.

\subsection{Light Source Positions}

We focus on characterizing the t-PBM treatment strategy. A pair of transcranial source configurations ${ }^{18}$ are investigated: (1) F3-F4: two light-emitting diode (LED) array sources are placed above and centered, respectively, at the F3 and F4 (10-20 EEG positions); (2) Fp1-FpzFp2: one LED array is placed on the forehead and between the Fp1-Fpz-Fp2 positions (referred to as the Fpz position hereinafter). The definitions of these source positions and orientations are similar to the study by Cassano et al., ${ }^{18}$ with the exception that the F3-F4 source arrays are rotated $\sim 90 \mathrm{deg}$, as shown in Fig. 3, to better align with the dIPFC region and maximize light delivery. The simulated LED arrays follow the dimensions and parameters of an available PBM source (Omnilux New-U, Photomedex, Horsham, Pennsylvania).

\subsection{Simulation Settings}

In our study, a total of 15 simulations, a combination of 3 source positions and 5 wavelengths $(670,810,850,980$, and $1064 \mathrm{~nm})$, are performed for each segmented brain atlas using our

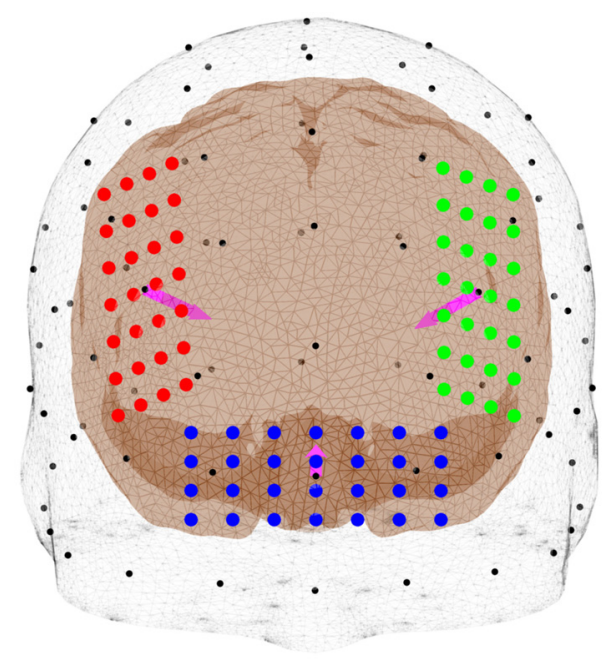

Fig. 3 Illustrations of source positions. Three extracranial source positions are shown-F3 (green), F4 (red), and Fpz (blue). Each source is an array of LEDs represented by colored dots. The magenta arrow represents the source direction. 
Table 1 The estimated optical properties of the ECT layer at five wavelengths. $\mu_{a}$-absorption coefficient $\left(\mathrm{mm}^{-1}\right), \mu_{s}-$ scattering coefficient $\left(\mathrm{mm}^{-1}\right), g$-anisotropy, and $n$-refractive index.

\begin{tabular}{lcccc}
\hline \hline Wavelength $(\mathrm{nm})$ & $\mu_{a}$ & $\mu_{s}$ & $g$ & $n$ \\
\hline 670 & 0.036 & 10.32 & 0.90 & 1.37 \\
810 & 0.021 & 11.22 & 0.89 & 1.37 \\
850 & 0.021 & 10.78 & 0.89 & 1.37 \\
980 & 0.031 & 9.79 & 0.89 & 1.37 \\
1064 & 0.033 & 9.35 & 0.89 & 1.37 \\
\hline \hline
\end{tabular}

graphics processing unit (GPU)-accelerated photon transport simulator (MCX). ${ }^{32,33}$ The optical properties of each layer (WM, GM, and CSF) are identical to those from our earlier study, ${ }^{18}$ with the exception that the optical properties of the ECT layer (Table 1) are derived using the weighted average of the properties for fat, muscle, skin tissue, and skull; the weights are derived from volume fractions of the respective tissue types in the Colin27 atlas. ${ }^{54,55}$ For simplicity, we assume the optical properties for each tissue type are independent of age. In all simulations, a total of $10^{9}$ photons are launched. To reduce the effect of shot noise in regions distal to the light source, an adaptive nonlocal mean filter is applied. ${ }^{56}$ All atlases share the same resolution of $1 \times 1 \times 1 \mathrm{~mm}^{3}$ isotropic voxels. Normalized average energy deposition $\left(\mathrm{J} / \mathrm{cm}^{3}\right)$, as described in the study by Cassano et al., ${ }^{18}$ is then computed for each atlas and compared across different age groups. The only exception is that peak fluence (99th percentile of the target) is adopted for computing the exposure duration for one treatment session.

\subsection{Extracerebral Tissues Thickness Estimation}

To further understand the age dependency of t-PBM light dosage, we also compute the thickness of the ECT layer. In Fig. 4, three ECT regions for F3 (green), F4 (red), and Fpz (blue) positions are created for computing the ECT thickness. The average ECT thicknesses are estimated by (1) using the "Iso2Mesh" toolbox ${ }^{57}$ to create meshes for the inner and outer surfaces of the ECT layer for each atlas, (2) projecting the illuminated areas from the outer surface inward along

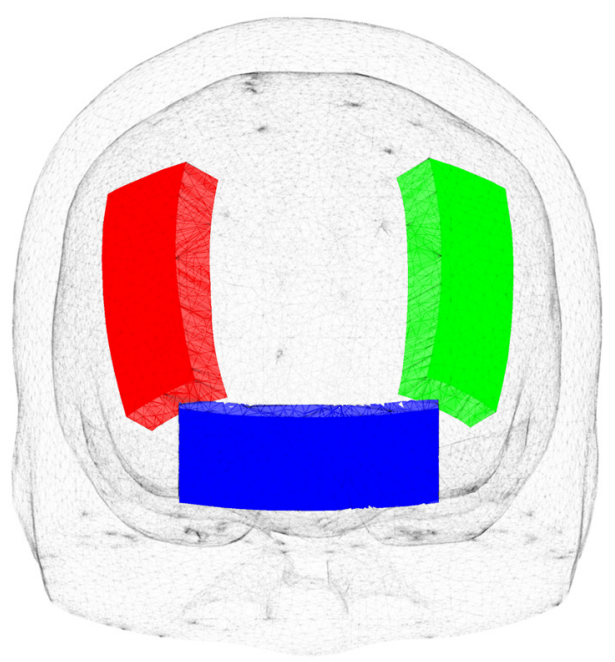

Fig. 4 The ECT regions used to compute the ECT thickness under F3 (green), F4 (red), and Fpz (blue) positions. 
the normal direction to generate a truncated volume, and (3) computing the average thickness by dividing the enclosed volume by the area of the illuminated area.

\section{Results}

\subsection{Photon Dosimetry Assessment}

Sample sagittal energy deposition maps are shown in Figs. 5(a)-5(c) to demonstrate qualitatively the changes of light distributions over age. The selected plots are generated using an Fpz source at $810 \mathrm{~nm}$ for three different age groups- 5,40 to 44 , and 85 to 89 years. In Fig. 6, we summarize the age-dependent average energy depositions in dIPFC and vmPFC using F3-F4 and Fpz positions, respectively, for five selected wavelengths. Similar to our previous findings, ${ }^{18}$ an 810-nm illumination appears to provide the highest energy deposition across a wide range of age spans, followed by 1064 and $850 \mathrm{~nm}$. In addition, a strong linear correlation on this log-scaled plot is found between there wavelengths. For extended vmPFC (not included in Fig. 6), the same conclusions can be drawn. For simplicity, we only report results at $810 \mathrm{~nm}$ hereinafter. In Fig. 7, we show the top five brain parcellations that have received the highest average energy deposition. The parcellations shown in the legend are roughly ranked in descending order based on energy deposition, although such orders may vary slightly across the age groups. The numbers in parentheses correspond to the numbering shown in Figs. 2(c) and 2(d).

In Fig. 8(a), we show the average energy deposition for dlPFC and vmPFC using both source positions. In Fig. 8(b), we show the exposure duration per session over age. Exposure durations ( $t$ in minute) are estimated to ensure that the peak fluence (99th percentile of the target ${ }^{18}$ ) at

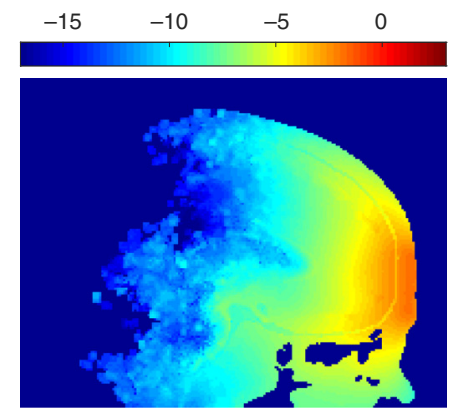

(a)

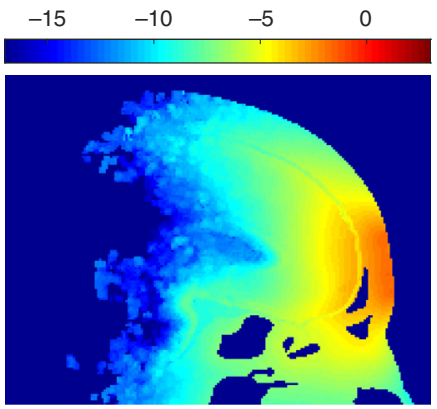

(b)

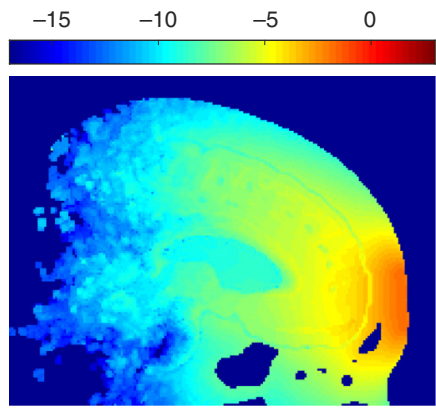

(c)

Fig. 5 Sample energy distributions. Energy deposition $\left(\mathrm{J} / \mathrm{cm}^{3}\right)$ in $\log _{10}$ scale for (a) 5 , (b) 40 to 44 , and (c) 85 to 89 years old. Only Fpz source position and 810-nm results are shown here.

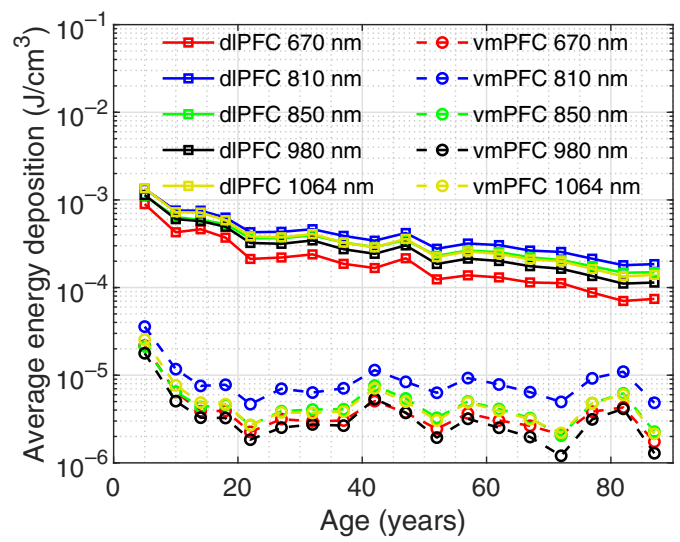

Fig. 6 Average energy deposition for five wavelengths across age. The plots for various wavelengths are shown in different colors. The solid line with square markers and the dashed line with circular markers indicate dIPFC using F3-F4 position and vmPFC using Fpz position, respectively. 


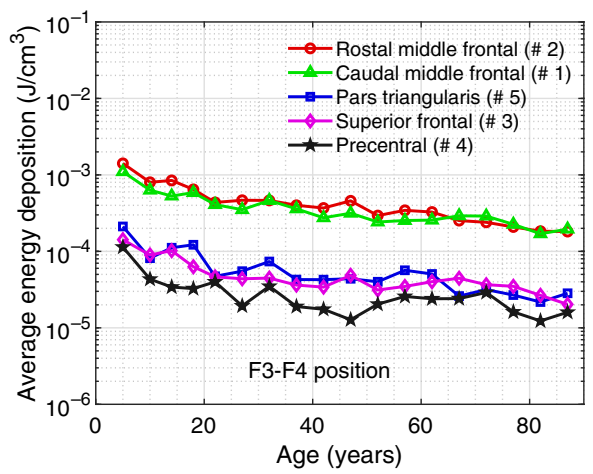

(a)

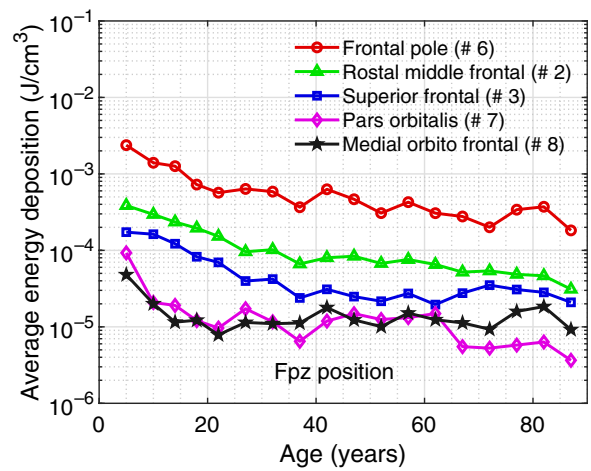

(b)

Fig. 7 Average energy deposition variations across age. We show results for (a) F3-F4 and (b) Fpz source positions. Each plot includes the parcellations with top five average energy deposition in descending order from top to bottom in the legend. The numbers in the parentheses correspond to the parcellation labels in Figs. 2(c) and 2(d). Only results from $810 \mathrm{~nm}$ are shown.

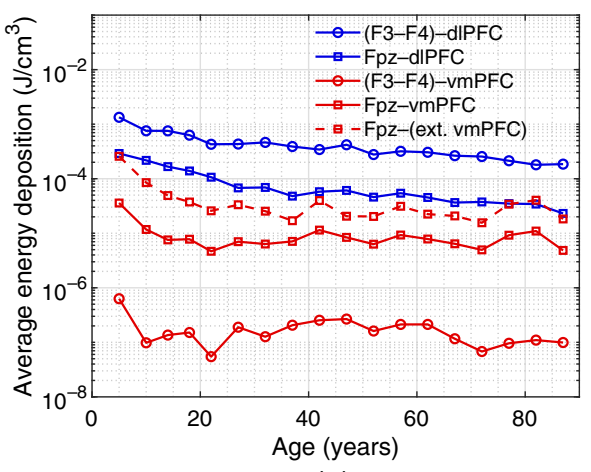

(a)

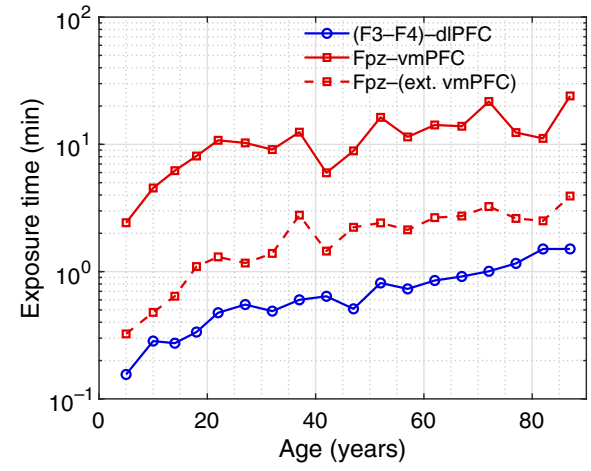

(b)

Fig. 8 Plots of (a) average energy deposition and (b) estimated treatment duration (in minute) for dIPFC and vmPFC. Each line represents the result for a source-target region pair, as listed in the legends. All results are computed at $810 \mathrm{~nm}$. The dashed lines represent the results for extended vmPFC (ext. vmPFC). To estimate exposure time, we assume that the skin irradiance is $300 \mathrm{~mW} / \mathrm{cm}^{2}$.

dIPFC and vmPFC reaches an optimal fluence of $3 \mathrm{~J} / \mathrm{cm}^{2}$ per session for F3-F4 and Fpz positions, respectively. It is noteworthy that, in our earlier study, ${ }^{18}$ a treatment session is effective when the upper quartile of the target reaches fluence of $3 \mathrm{~J} / \mathrm{cm}^{2}$, whereas the 99 th percentile of the target is used in this paper. The revision is made to minimize the light fluence on the skin and reduce the risk of overexposure. Furthermore, the engagement by t-PBM of the most superficial cortex, for any given brain area, is considered sufficient to modulate the emotion regulation circuitry. The expression of $t$ (in seconds) can be written as

$$
t=\phi_{e} V \mu_{a} /\left(k E_{s} A\right),
$$

where $\phi_{e}\left(\mathrm{~J} / \mathrm{cm}^{2}\right)$ is the effective fluence achieved at the target region of interest (ROI), $V\left(\mathrm{~cm}^{3}\right)$ is the volume with energy deposition $>99$ th percentile inside the ROI, $\mu_{a}\left(\mathrm{~cm}^{-1}\right)$ is the ROI absorption coefficient, $k \in[0,1]$ is the percentage of the source energy delivered to the volume with energy deposition $>99$ th percentile inside the ROI, $E_{s}\left(\mathrm{~W} / \mathrm{cm}^{2}\right)$ is the skin irradiance of the source, and $A\left(\mathrm{~cm}^{2}\right)$ is the size of the illumination area. The skin irradiance is set at 300 or $30 \mathrm{~mW} / \mathrm{cm}^{2}$ for computing the exposure duration. The illumination area is 28.4 and $28.4 \times$ $2 \mathrm{~cm}^{2}$ for Fpz and F3-F4 positions, respectively, based on the simulated source device. ${ }^{18}$ 


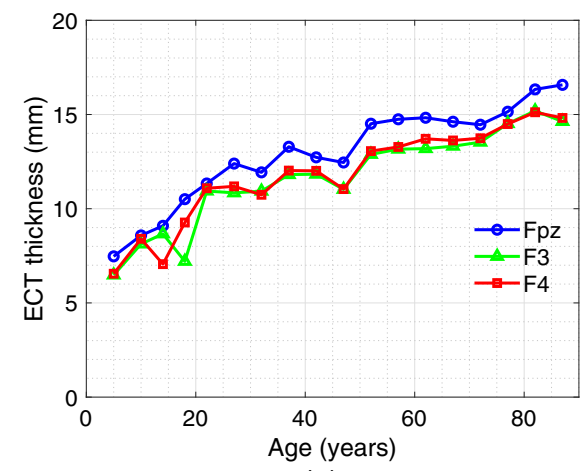

(a)

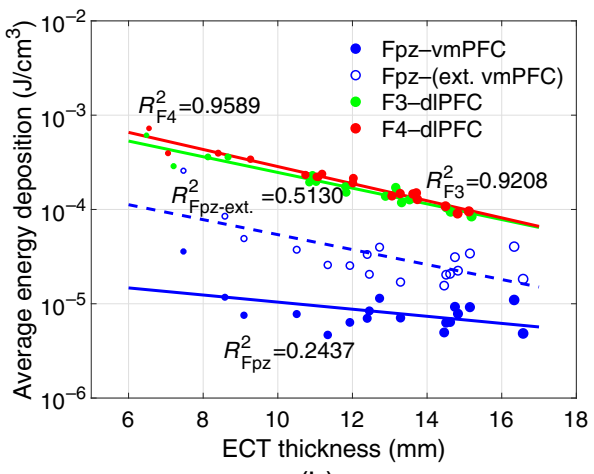

(b)

Fig. 9 (a) The relationship between age and thickness of ECT. (b) The correlation between thickness of ECT and average energy deposition (in $\log _{10}$ scale) in the dIPFC and vmPFC. For F3 and F4 positions, the target region is dIPFC, whereas for Fpz position, the target region is vmPFC/ extended vmPFC (ext. vmPFC). The size of the markers represents the age group.

In Fig. 9(a), we plot the ECT thicknesses over age groups for F3, F4, and Fpz positions. The correlations between the ECT thickness and the average energy deposition (in $\log _{10}$ scale) are demonstrated in Fig. 9(b) in the target region using the 810-nm illumination. The target region is dlPFC for F3/F4 placement and vmPFC/extended vmPFC for Fpz source placement. In our results, due to the low thickness and low absorption, the CSF layer shows minor effects on energy deposition across age compared to the ECT layer. Thus, the CSF layer is not considered in Fig. 9.

\section{Discussions}

From the sample energy deposition maps in Fig. 5, we can visually observe that light penetration to the brain decreases as age increases. The increasing brain size and ECT layer thickness can also be observed in Fig. 5, which impedes energy delivery to the desired brain tissues.

In Fig. 6, all wavelengths show an overall decreasing energy deposition as increasing age for both dIPFC and vmPFC. The linear correlation coefficients between $810 \mathrm{~nm}$ and other tested wavelengths are found to be $>0.99$ for both ROIs, suggesting that the age variation has a weak dependency on wavelength. Comparing between wavelengths, 810-nm wavelength delivers the highest energy deposition; 850- and 1064-nm wavelengths deliver more energy than 670- and $980-\mathrm{nm}$ wavelengths in most cases. These findings generally agree with previously published simulation-based studies, ${ }^{18,58}$ however, we want to indicate that there is a wide range of brain optical properties in literature, due to diverse measurement techniques and experimental settings. Different choices of literature values could potentially lead to different rankings in wavelength efficiency. ${ }^{58,59}$

Figure 7(a) shows that the top five regions in energy deposition are mostly located in the frontal lobe of the brain, which also includes the dIPFC. The rostral middle frontal gyrus and the caudal middle frontal gyrus that compose the dIPFC receive $\sim 10$-fold more energy deposition than the remaining three. This is mainly a result of shorter distances between these two regions and the source when compared to other parcellations. In Fig. 7(b), the energy deposition in the frontal pole region is over 10-fold higher than that of other parcellations. Furthermore, the first four parcellations are not part of vmPFC. This is due to the fact that the vmPFC is located at the bottom of cerebral hemispheres, as shown in Fig. 2(b), while the Fpz source position delivers light directly toward the frontal pole. Across all age groups, the F3-F4 position delivers $0.23 \%$ to $2.9 \%$ of the total energy into dIPFC, whereas the Fpz position only delivers $0.0066 \%$ to $0.09 \%$ of the total energy into vmPFC. Based on this result, the Fpz position appears to be less effective in delivering light to the target region in comparison with the F3-F4 position. In both Figs. 7(a) and 7(b), we can observe a decrease in energy deposition, as age increases, similar to the overall trend shown in Fig. 6. 
In Fig. 8(a), a decrease in energy deposition can again be found for all source-region pairs as age increases. However, energy deposition at the vmPFC shows a stronger decrease in younger age groups than that at the dlPFC. For example, the energy deposition decreases by $86.97 \%$ and $67.98 \%$ from 5 to 20 years old for Fpz-vmPFC and (F3-F4)-dlPFC, respectively. In addition, during adulthood, the vmPFC has a slower decay rate in energy deposition along age than the dIPFC. With frontal pole included, the extended vmPFC has an average fourfold increase in energy deposition across age groups compared to the vmPFC. Therefore, the actual energy deposition at the vmPFC may vary depending on the definition of the target region. Furthermore, the energy deposition of Fpz-dIPFC is generally higher than that of Fpz-vmPFC as well as Fpz-(extended vmPFC) across age, which is caused by the location of vmPFC illustrated previously. This suggests that an effective t-PBM treatment targeting at vmPFC/extended vmPFC with an Fpz source also delivers sufficient dosage to dlPFC, but not vice versa.

The plots in Fig. 8(b) show that the desired treatment duration increases with age for both (F3-F4)-dlPFC and Fpz-vmPFC treatments. For (F3-F4)-dlPFC with skin irradiance of $300 \mathrm{~mW} / \mathrm{cm}^{2}$, the effective fluence can be achieved in $<2 \mathrm{~min}$ across all ages. However, the exposure duration is much longer for Fpz-vmPFC and is $\sim 10$ to 20 min after 20 years of age. This is due to insufficient energy deposition at the vmPFC as discussed earlier. The addition of frontal pole to the extended vmPFC reduces the exposure duration by $71 \%$ to $88 \%$ compared to the vmPFC since the Fpz position mostly concentrates energy at the frontal pole. Caution should be exercised when applying these findings to clinical research or practice. It is in fact unusual to expose the skin for up to $20 \mathrm{~min}$ to high irradiance of $300 \mathrm{~mW} / \mathrm{cm}^{2}$. Our data also suggest the potential for overexposure of the most superficial brain areas, such as the frontal poles when the light source is positioned near Fpz. Furthermore, there may be other treatment strategies with different skin irradiance. From Eq. (1), we can see that, given a fixed illumination area, the factors that determine the treatment exposure duration are the effective fluence and irradiance. In addition, the exposure duration is inversely proportional to the skin irradiance. Therefore, we can adjust the exposure duration by increasing or decreasing the skin irradiance.

In Fig. 9(a), we notice that for all F3, F4, and Fpz positions, the thickness of the ECT layer increases as age increases. The ECT regions under the F3 and F4 positions have very similar thickness values across the age due to symmetry. In comparison, the ECT thickness for Fpz position is generally larger than the other two, which coincides with the findings reported previously. ${ }^{60}$

In Fig. 9(b), the plots between the ECT thickness and the log-scaled energy deposition show a rough linear relationship between the two parameters. Applying linear regressions, we obtained four linear models for (1) Fpz-vmPFC: $y=-0.0376 x-4.6061\left(R^{2}=0.2437\right)$, (2) Fpz(extended vmPFC): $y=-0.0794 x-3.4733\left(R^{2}=0.5130\right)$, (3) F3-dlPFC: $y=-0.0833 x-$ $2.7749\left(R^{2}=0.9208\right)$, and (4) F4-dlPFC: $y=-0.0906 x-2.6388\left(R^{2}=0.9589\right)$. For both F3 and F4 positions, the ECT thicknesses show a strong negative linear correlation to the average energy deposition in log scale at the dlPFC. The plots in Fig. 9(b) show discernible deviations from a linear fit in younger ages, possible due to the boundary effect in smaller-sized head models. Only a weak linear correlation is found for Fpz position. It is our belief that the relatively weak correlation at the Fpz source is a result of larger separation between the target region (vmPFC) and the source. Nonetheless, an overall decreasing trend is evident for the vmPFC energy deposition from the Fpz-centered source. For Fpz sources targeting the extended vmPFC, the result presents a stronger linear correlation compared to Fpz-vmPFC. The frontal pole merged within the extended vmPFC is closer to the Fpz position and shows a strong linear correlation $\left(R^{2}=0.8660\right)$, which raises the overall correlation for the Fpz-(extended vmPFC). These results indicate that the anatomical development of the head, and especially the increase of ECT thickness, is largely responsible for decreased energy deposition in the brain as a result of growth and aging. Furthermore, shorter distances between the source position and target region result in greater correlation between energy deposition and ECT thickness.

In addition, we have repeated our simulations using refined brain anatomical models by further separating the ECT layer into the scalp and skull layers using FSL. Our simulations using such atlases show very similar results (not included) to our above findings. This suggests that the overall ECT tissue thickness plays a more important role in t-PBM treatment compared to the individual scalp/skull thicknesses. 


\section{Conclusions and Summary}

In this report, we systematically investigated light dosimetry in the t-PBM treatment across a wide span of ages. To ensure accurate quantification, we used anatomically appropriate brain atlases and state-of-the-art MC simulations. For modeling the brain anatomy, we have developed a robust workflow to create multilayered head segmentations from publicly available Neurodevelopmental MRI atlas library data. For each brain segmentation, we have also generated corresponding brain parcellations to facilitate quantitative analysis. A number of conclusions have been found from our simulation results, and many of those are consistent with our previous study. ${ }^{18}$ First, the energy deposition decreases across lifespan regardless of the source position and parcellations. However, the decline is faster before adulthood and this is more noticeable for the vmPFC. Second, wavelength selection shows a negligible impact to the trend of energy deposition decay over age, despite that the 810 -nm wavelength consistently gives the highest energy deposition compared to four other commonly used wavelengths. Although this result is generally consistent with previous simulation-based studies, ${ }^{13,61}$ we would like to highlight that our results are dependent on the choices of brain optical properties. As discussed in the study by Cassano et al., ${ }^{18}$ there is no widely accepted set of optical properties for brain tissues. Using values from different literature may lead to different preferences in wavelength. ${ }^{59}$ Third, a negative linear correlation is observed between the thickness of ECT layer and the log-scale average energy deposition in two selected brain ROIs, suggesting that the brain anatomical changes are largely responsible for the observed age-dependent variations.

Furthermore, the Fpz source position has longer separation distances from the vmPFC region compared to the separations between the dlPFC and the F3-F4 source, suggesting that higher source intensity (alternatively, longer exposure time) is required when targeting the vmPFC region compared to the dlPFC region. In addition, the frontal pole [label 6 in Fig. 2(d)] shows stronger energy deposition compared to the remaining parts of vmPFC. Thus, the exposure duration for the extended vmPFC (vmPFC + frontal pole) is shortened. In addition, we provide a simple approach to estimate the treatment duration using our simulated results. From this relationship, the exposure duration generally increases as age increases. These quantitative assessments are expected to provide clinicians the guidance in designing personalized PBM treatment plans and ultimately improve the outcome of the procedures.

We would like to mention that there are several known limitations in this study. First, the simulations are performed using averaged brain atlases derived from public datasets. We acknowledge that using subject-specific brain segmentations may lead to more accurate estimations. In fact, our reported processing methods can be directly applied to subject-specific anatomical scans if such data become available, making it suitable for personalized t-PBM treatment planning. Second, the brain optical properties are assumed to be static across age groups. Although optical property measurements of age-dependent brain tissue are generally lacking, earlier studies on breast tissues have observed little or no correlation with age. ${ }^{62,63}$ A correlation between dermis tissue absorption over age was reported, ${ }^{64,65}$ but the magnitude of such variation is relatively small. Our analyses can be easily extended to include age-dependent optical properties when such data become available in the future. Third, this study is specifically focused on treating MDD by performing t-PBM to target vmPFC and dIPFC brain regions. If modeling other t-PBM source forms and brain ROIs becomes necessary, we can apply the same multilayered brain segmentation and parcellation models to quickly recreate the results for the new targeted regions. Finally, we largely rely on dedicated brain segmentation tools, namely, FSL and FreeSurfer, to create the brain anatomical models used in this study. The choice of neuroanatomical analysis tools may lead to variations in brain segmentations and simulation results. In the next steps, we may extend this study to use more accurate mesh-based $\mathrm{MC}^{66,67}$ simulations and subject-specific scans.

\section{Disclosures}

Dr. Cassano's salary was supported by the Harvard Psychiatry Department (Dupont Warren Fellowship and Livingston Award), the Brain and Behavior Research Foundation (NARSAD Young Investigator Award), and an unrestricted grant from Photothera Inc. Drug donation was 
received from Teva. Travel reimbursement was received from Pharmacia and Upjohn. He has received consultation fees from Janssen Research and Development. He has also filed several patents related to the use of NIR light in psychiatry. PhotoMedex, Inc. supplied four devices for a clinical study. He has recently received unrestricted funding from LiteCure, Inc., to conduct a study on t-PBM for the treatment of MDD and to conduct a study on healthy subjects. He cofounded a company (Niraxx Light Therapeutics) focused on the development of new modalities of treatment based on NIR light; he is a consultant for the same company. He also received funding from Cerebral Sciences to conduct a study on t-PBM for generalized anxiety disorder. No conflicts of interest, financial or otherwise, are declared by the other authors.

\section{Acknowledgments}

This work was supported by the National Institutes of Health (NIH) under Grant Nos. R01GM114365, R01-CA204443, and R01-EB026998. The authors would like to thank Dr. Lilla Zöllei for her inputs regarding FreeSurfer software and Edward Xu for his help on developing the paper.

\section{References}

1. N. Hoertel et al., "Why does the lifetime prevalence of major depressive disorder in the elderly appear to be lower than in younger adults? Results from a national representative sample," J. Affect. Disord. 149(1-3), 160-165 (2013).

2. B. Birmaher, C. Arbelaez, and D. Brent, "Course and outcome of child and adolescent major depressive disorder," Child Adolesc. Psychiatr. Clin. N. Am. 11(3), 619-637 (2002).

3. B. Birmaher et al., "Practice parameter for the assessment and treatment of children and adolescents with depressive disorders," J. Am. Acad. Child Adolesc. Psychiatry 46(11), 1503-1526 (2007).

4. D. Sobieraj et al., "Adverse effects of pharmacologic treatments of major depression in older adults," J. Am. Geriatr. Soc. 67(8), 1571-1581 (2019).

5. M. Olfson, C. Blanco, and S. C. Marcus, "Treatment of adult depression in the United States," JAMA Intern. Med. 176(10), 1482-1491 (2016).

6. M. Sinyor, A. Schaffer, and A. Levitt, "The sequenced treatment alternatives to relieve depression (STAR*D) trial: a review," Can. J. Psychiatry 55(3), 126-135 (2010).

7. P. Cassano and M. Fava, "Tolerability issues during long-term treatment with antidepressants," Ann. Clin. Psychiatry 16(1), 15-25 (2004).

8. I. van Beljouw et al., "Reasons and determinants for not receiving treatment for common mental disorders," Psychiatr. Serv. 61(3), 250-257 (2010).

9. F. Salehpour et al., "Brain photobiomodulation therapy: a narrative review," Mol. Neurobiol. 55(8), 6601-6636 (2018).

10. M. A. Caldieraro and P. Cassano, "Transcranial and systemic photobiomodulation for major depressive disorder: a systematic review of efficacy, tolerability and biological mechanisms," J. Affect. Disord. 243, 262-273 (2018).

11. P. Cassano et al., "Review of transcranial photobiomodulation for major depressive disorder: targeting brain metabolism, inflammation, oxidative stress, and neurogenesis," Neurophotonics 3(3), 031404 (2016).

12. J. R. Jagdeo et al., "Transcranial red and near infrared light transmission in a cadaveric model," PLoS One 7(10), e47460 (2012).

13. T. A. Henderson and L. D. Morries, "Near-infrared photonic energy penetration: can infrared phototherapy effectively reach the human brain?" Neuropsychiatr. Dis. Treat. 11, 2191-2208(2015)

14. M. R. Hamblin and T. N. Demidova, "Mechanisms of low level light therapy," Proc. SPIE 6140, 614001 (2006).

15. M. R. Hamblin, "Shining light on the head: photobiomodulation for brain disorders," $B B A$ Clin. 6, 113-124 (2016). 
16. Y. Chen et al., "Thermal effects of transcranial near-infrared laser irradiation on rabbit cortex," Neurosci. Lett. 553, 99-103 (2013).

17. P. Cassano et al., "Transcranial photobiomodulation for the treatment of major depressive disorder. The ELATED-2 pilot trial," Photomed. Laser Surg. 36(12), 634-646 (2018).

18. P. Cassano et al., "Selective photobiomodulation for emotion regulation: model-based dosimetry study," Neurophotonics 6(1), 015004 (2019).

19. A. Adeloye, K. R. Kattan, and F. N. Silverman, "Thickness of the normal skull in the American Blacks and Whites," Am. J. Phys. Anthropol. 43(1), 23-30 (1975).

20. V. Sabancoğullar et al., "Diploe thickness and cranial dimensions in males and females in mid-Anatolian population: an MRI study," Forensic Sci. Int. 219(1-3), 289.e1-289.e7 (2012).

21. H. Hori et al., "The thickness of human scalp: normal and bald," J. Invest. Dermatol. 58(6), 396-399 (1972).

22. J. E. Richards et al., "A database of age-appropriate average MRI templates," NeuroImage 124, 1254-1259 (2016).

23. P. T. Fillmore, M. C. Phillips-Meek, and J. E. Richards, "Age-specific MRI brain and head templates for healthy adults from 20 through 89 years of age," Front. Aging Neurosci. 7, 44 (2015).

24. C. E. Sanchez, J. E. Richards, and C. R. Almli, "Age-specific MRI templates for pediatric neuroimaging," Dev. Neuropsychol. 37(5), 379-399 (2012).

25. F. Shi et al., "Infant brain atlases from neonates to 1- and 2-year-olds," PLoS One 6(4), e18746 (2011).

26. C. Zhu and Q. Liu, "Review of Monte Carlo modeling of light transport in tissues," J. Biomed. Opt. 18(5), 050902 (2013).

27. A. Corlu et al., "Three-dimensional in vivo fluorescence diffuse optical tomography of breast cancer in humans," Opt. Express 15(11), 6696-6716 (2007).

28. Q. Fang et al., "Combined optical and x-ray tomosynthesis breast imaging," Radiology 258(1), 89-97 (2011).

29. A. H. Hielscher, R. E. Alcouffe, and R. L. Barbour, "Comparison of finite-difference transport and diffusion calculations for photon migration in homogeneous and heterogeneous tissues," Phys. Med. Biol. 43(5), 1285-1302 (1998).

30. A. Custo et al., "Effective scattering coefficient of the cerebral spinal fluid in adult head models for diffuse optical imaging," Appl. Opt. 45(19), 4747-4755 (2006).

31. S. L. Jacques and L. Wang, "Monte Carlo modeling of light transport in tissues," in OpticalThermal Response of Laser-Irradiated Tissue, A. J. Welch and M. J. C. van Gemert, Ed., pp. 73-100, Springer, Boston, Massachusetts (1995).

32. Q. Fang and D. A. Boas, "Monte Carlo simulation of photon migration in $3 \mathrm{D}$ turbid media accelerated by graphics processing units," Opt. Express 17(22), 20178-20190 (2009).

33. L. Yu et al., "Scalable and massively parallel Monte Carlo photon transport simulations for heterogeneous computing platforms," J. Biomed. Opt. 23(1), 010504 (2018).

34. C. D. Good et al., "A voxel-based morphometric study of ageing in 465 normal adult human brains," NeuroImage 14(1), 21-36 (2001).

35. C. D. Smith et al., "Age and gender effects on human brain anatomy: a voxel-based morphometric study in healthy elderly," Neurobiol. Aging 28(7), 1075-1087 (2007).

36. S. M. Smith, "Fast robust automated brain extraction," Hum. Brain Mapp. 17(3), 143-155 (2002).

37. C. E. Sanchez, J. E. Richards, and C. R. Almli, "Neurodevelopmental MRI brain templates for children from 2 weeks to 4 years of age," Dev. Psychobiol. 54(1), 77-91 (2012).

38. S. Brigadoi et al., "A 4D neonatal head model for diffuse optical imaging of pre-term to term infants," NeuroImage 100, 385-394 (2014).

39. A. R. Groves et al., "Benefits of multi-modal fusion analysis on a large-scale dataset: lifespan patterns of inter-subject variability in cortical morphometry and white matter microstructure," NeuroImage 63(1), 365-380 (2012).

40. P. A. Yushkevich et al., "User-guided 3D active contour segmentation of anatomical structures: significantly improved efficiency and reliability," NeuroImage 31(3), 1116-1128 (2006). 
41. D. Arthur and S. Vassilvitskii, "k-means++: the advantages of careful seeding," in Proc. Eighteenth Annu. ACM-SIAM Symp. Discrete Algorithms, Society for Industrial and Applied Mathematics, pp. 1027-1035 (2007).

42. G. Auzias, O. Coulon, and A. Brovelli, "MarsAtlas: a cortical parcellation atlas for functional mapping," Hum. Brain Mapp. 37(4), 1573-1592 (2016).

43. A. D. Boes et al., "Right ventromedial prefrontal cortex: a neuroanatomical correlate of impulse control in boys," Soc. Cognit. Affect. Neurosci. 4(1), 1-9 (2008).

44. A. Powers et al., "Neural correlates and structural markers of emotion dysregulation in traumatized civilians," Soc. Cognit. Affect. Neurosci. 12(5), 823-831 (2017).

45. R. A. Morey et al., "Amygdala, hippocampus, and ventral medial prefrontal cortex volumes differ in maltreated youth with and without chronic posttraumatic stress disorder," Neuropsychopharmacology 41(3), 791-801 (2016).

46. P. Nopoulos et al., "Hyperactivity, impulsivity, and inattention in boys with cleft lip and palate: relationship to ventromedial prefrontal cortex morphology," J. Neurodev. Disord. 2(4), 235-242 (2010).

47. R. S. Desikan et al., "An automated labeling system for subdividing the human cerebral cortex on MRI scans into gyral based regions of interest," NeuroImage 31(3), 968-980 (2006).

48. B. Fischl, "FreeSurfer," NeuroImage 62(2), 774-781 (2012).

49. C. Destrieux et al., "Automatic parcellation of human cortical gyri and sulci using standard anatomical nomenclature," NeuroImage 53(1), 1-15 (2010).

50. A. M. Fjell et al., "Multimodal imaging of the self-regulating developing brain," Proc. Natl. Acad. Sci. U. S. A. 109(48), 19620-19625 (2012).

51. S. Ehrlich et al., "The COMT Val108/158Met polymorphism and medial temporal lobe volumetry in patients with schizophrenia and healthy adults," NeuroImage 53(3), 992-1000 (2010).

52. E. C. Finger et al., "Abnormal ventromedial prefrontal cortex function in children with psychopathic traits during reversal learning," Arch. Gen. Psychiatry 65(5), 586-594 (2008).

53. R. C. Wolf et al., "Emotion recognition deficits associated with ventromedial prefrontal cortex lesions are improved by gaze manipulation," Cortex 82, 255-262 (2016).

54. D. L. Collins et al., "Design and construction of a realistic digital brain phantom," IEEE Trans. Med. Imaging 17(3), 463-468 (1998).

55. C. J. Holmes et al., "Enhancement of MR images using registration for signal averaging," J. Comput. Assist. Tomogr. 22(2), 324-333 (1998).

56. Y. Yuan et al., "Graphics processing units-accelerated adaptive nonlocal means filter for denoising three-dimensional Monte Carlo photon transport simulations," J. Biomed. Opt. 23(12), 121618 (2018).

57. Q. Fang and D. A. Boas, "Tetrahedral mesh generation from volumetric binary and grayscale images," in IEEE Int. Symp. Biomed. Imaging: From Nano to Macro, IEEE, pp. 1142-1145 (2009).

58. P. Wang and T. Li, "Which wavelength is optimal for transcranial low-level laser stimulation?" J. Biophotonics 12(2), e201800173 (2019).

59. X. Wang et al., "Up-regulation of cerebral cytochrome-c-oxidase and hemodynamics by transcranial infrared laser stimulation: a broadband near-infrared spectroscopy study," J. Cereb. Blood Flow Metab. 37(12), 3789-3802 (2017).

60. G. E. Strangman, Q. Zhang, and Z. Li, "Scalp and skull influence on near infrared photon propagation in the Colin27 brain template," NeuroImage 85, 136-149 (2014).

61. F. Salehpour et al., "Penetration profiles of visible and near-infrared lasers and light-emitting diode light through the head tissues in animal and human species: a review of literature," Photobiomodul. Photomed. Laser Surg. 37(10), 581-595 (2019).

62. T. L. Troy, D. L. Page, and E. M. Sevick-Muraca, "Optical properties of normal and diseased breast tissues: prognosis for optical mammography," J. Biomed. Opt. 1(3), 342-356 (1996).

63. T. Durduran et al., "Bulk optical properties of healthy female breast tissue," Phys. Med. Biol. 47(16), 2847-2861 (2002).

64. N. Bosschaart et al., "Optical properties of neonatal skin measured in vivo as a function of age and skin pigmentation," J. Biomed. Opt. 16(9), 097003 (2011). 
65. T. L. Troy and S. N. Thennadil, "Optical properties of human skin in the near infrared wavelength range of 1000 to $2200 \mathrm{~nm}$," J. Biomed. Opt. 6(2), 167-177 (2001).

66. Q. Fang, "Mesh-based Monte Carlo method using fast ray-tracing in Plücker coordinates," Biomed. Opt. Express 1(1), 165-175 (2010).

67. S. Yan, A. P. Tran, and Q. Fang, "Dual-grid mesh-based Monte Carlo algorithm for efficient photon transport simulations in complex three-dimensional media," J. Biomed. Opt. 24(2), 020503 (2019).

Yaoshen Yuan is a doctoral candidate at Northeastern University. He received his BE degree from Southeast University, China, in 2014 and MSE degree from Tufts University in 2016. His research interests include Monte Carlo simulation for photon transport, GPU algorithm enhancement, and signal processing.

Paolo Cassano, MD, $\mathrm{PhD}$, is a principal investigator at the Center for Anxiety and Traumatic Stress Disorders and the Depression Clinical and Research Program, Massachusetts General Hospital, and also an assistant professor at Harvard Medical School. He is specialized in photomodulation for psychiatric disorders. In 2015, his work on photomodulation was featured by The Boston Globe as one of the breakthroughs for the toughest medical conditions. He was also interviewed by CNN and by The Washington Post.

Matthew Pias is a graduate student at Northeastern University. He received his MS degree from Northeastern University in 2019 and his BSE degree from the University of Connecticut in 2017. His research interests include image processing and biomedical optics.

Qianqian Fang, $\mathrm{PhD}$, is currently an assistant professor in the Department of Bioengineering, Northeastern University, Boston, USA. He received his PhD from Dartmouth College in 2005. He then joined Massachusetts General Hospital and became an assistant professor in 2012, before he joined Northeastern University in 2015. His research interests include translational medical imaging systems, low-cost point-of-care devices for resource-limited regions, and high-performance computing tools to facilitate the development of next-generation imaging platforms. 